\title{
Nematode sperm motility ${ }^{*}$
}

\author{
Harold E. Smith ${ }^{\S}$
}

National Institute for Diabetes and Digestive and Kidney Diseases, National Institutes of Health, Bethesda MD, USA

\section{Table of Contents}

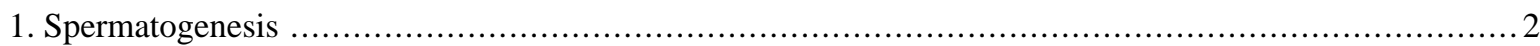

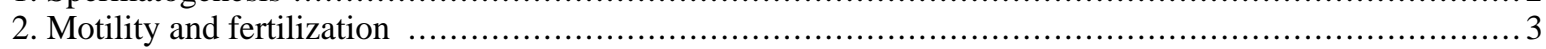

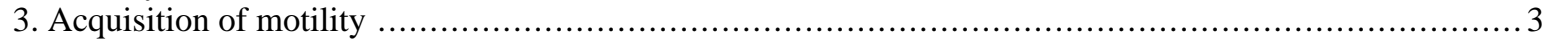

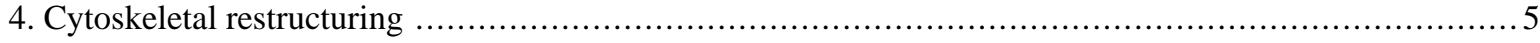

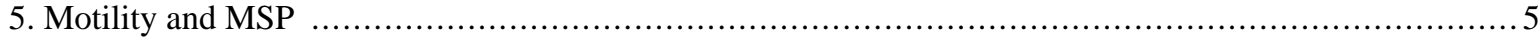

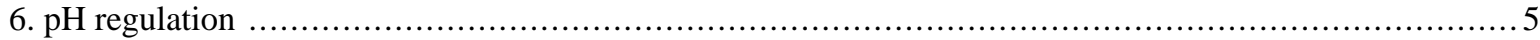

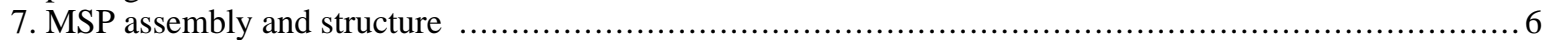

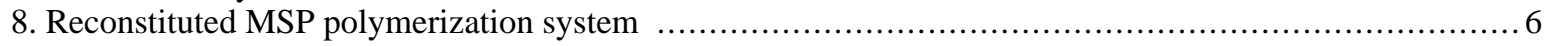

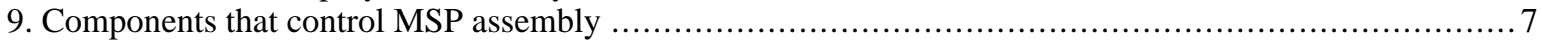

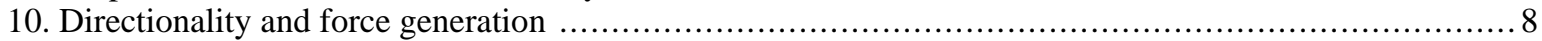

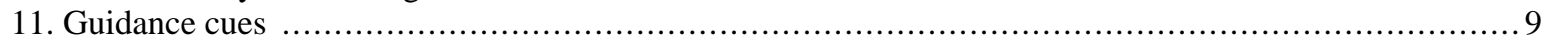

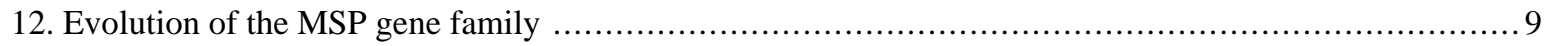

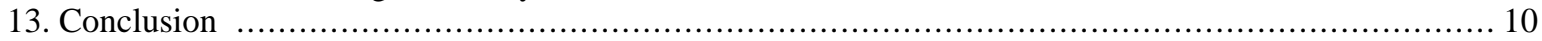

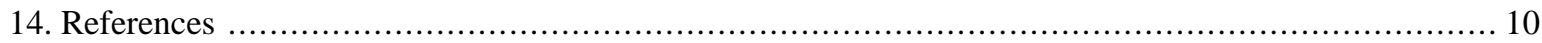

\begin{abstract}
Form follows function, and this maxim holds particularly true for the nematode sperm cell. Motility is essential for fertilization, and the process of spermatogenesis culminates in the production of a crawling spermatozoon with an extended pseudopod. However, the morphological similarity to amoeboid cells of other organisms is not conserved at the molecular level. Instead of utilizing the actin cytoskeleton and motor proteins, the pseudopod moves via the regulated assembly and disassembly of filaments composed of the major sperm protein (MSP). The current work reviews the structure and dynamics of MSP filament formation, the critical role of $\mathrm{pH}$ in MSP assembly, and the components that regulate this process. The combination of cytological, biochemical, and genetic approaches in this relatively simple system make nematode sperm an attractive model for investigating the mechanics of amoeboid cell motility.
\end{abstract}

\footnotetext{
*Edited by Michel Labouesse and Donald G. Moerman. Last revised September 17, 2013. Published April 4, 2014. This chapter should be cited as: Smith H.E. Nematode sperm motility (April 4, 2014), WormBook, ed. The C. elegans Research Community, WormBook, doi/10.1895/wormbook.1.68.2, http://www.wormbook.org.

Copyright: (C) 2014 Harold E. Smith. This is an open-access article distributed under the terms of the Creative Commons Attribution License, which permits unrestricted use, distribution, and reproduction in any medium, provided the original author and source are credited.

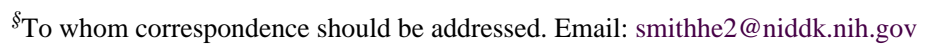




\section{Spermatogenesis}

Detailed descriptions of sperm development, or spermatogenesis, can be found in the WormBook chapter Spermatogenesis and elsewhere (e.g., Chu and Shakes, 2013); however, a brief summary is included here and illustrated in Figure 1 to emphasize the particulars of MSP assembly and segregation. Germline stem cells give rise to primary spermatocytes. The first meiotic division produces two secondary spermatocytes, and the second meiosis yields four haploid spermatids. The round, non-motile spermatids bud from the surface of the residual body, which contains components that are not needed for further development or sperm function (Ward et al., 1981). An extracellular signal induces the process of activation (detailed in Section 3) and causes pseudopod extension and motility that is the hallmark of mature spermatozoa (Figures 2 and 3).

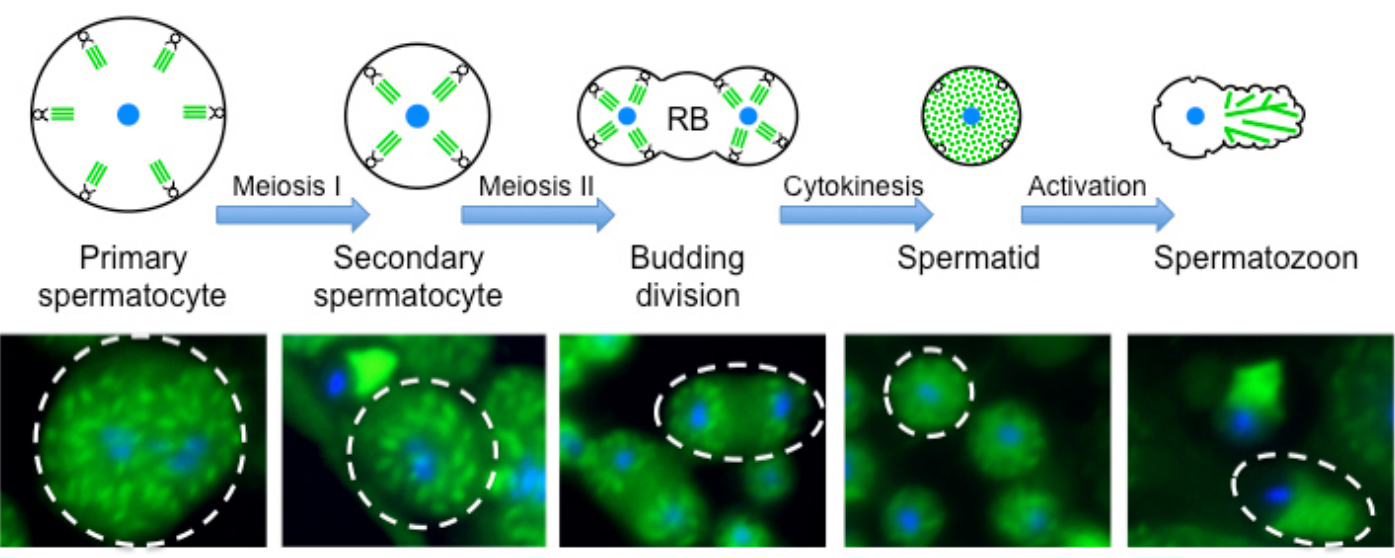

Figure 1. MSP distribution during sperm cell development. Top, schematic diagram; bottom, photomicrographs of sperm labeled with MSP-specific antibodies. In primary spermatocytes, MSP (green) begins to assemble as paracrystalline arrays in fibrous bodies associated with the membranous organelles (FB-MOs). Assembly continues through the secondary spermatocyte stage. FB-MOs segregate into spermatids during the budding division. After spermatids separate from the residual body (RB) during cytokinesis, MSP dissociates into the cytosol. Upon activation, MSP reassembles into filamentous fibers in the pseudopod. Images by Chris Uyehara and Diane Shakes.

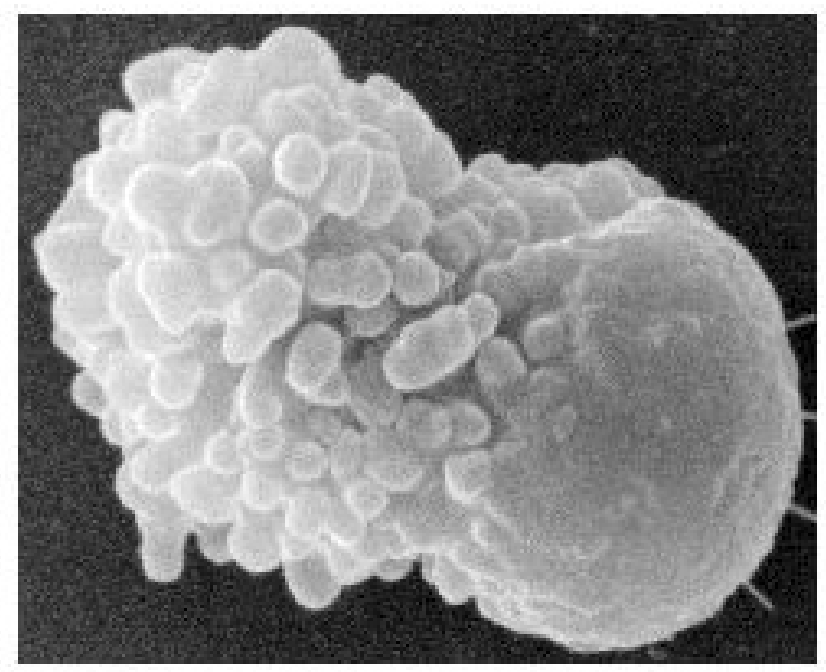

Figure 2. Scanning electron micrograph of spermatozoon. The cell body is to the right, and the numerous finger-like projections of the pseudopod to the left. Image courtesy of Sam Ward. 


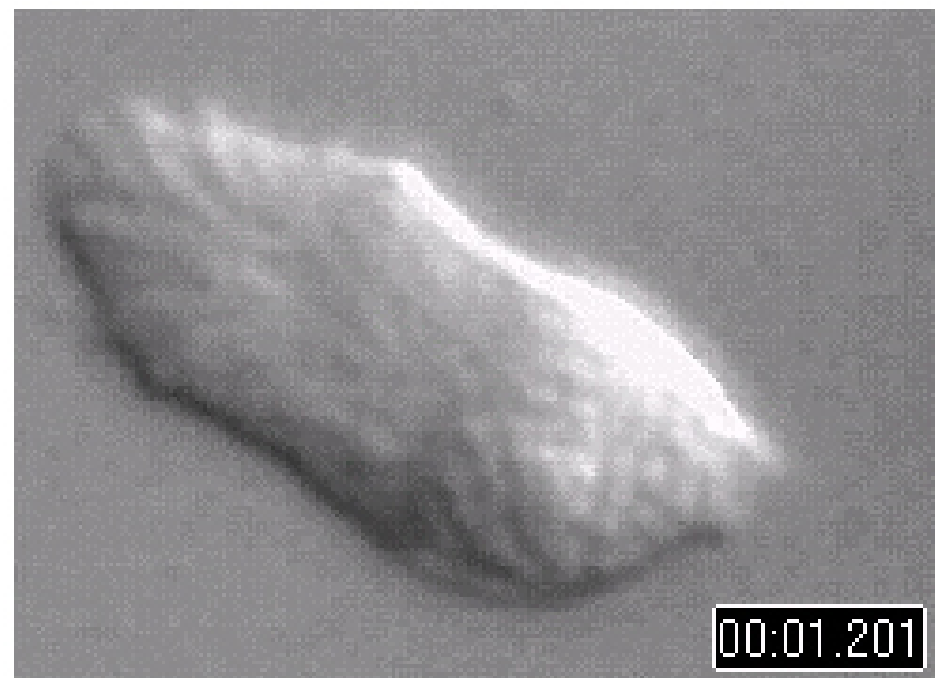

Figure 3. Video micrograph of pseudopod treadmilling during motility. The orientation is similar to Figure 2, with the pseudopod toward the upper left. New projections form at the leading edge of the pseudopod and flow back toward the cell body. Video by Paul Muhlrad.

MSP localization reflects the various stages of spermatogenesis (Ward and Klass, 1982; Roberts et al., 1986). Monoclonal antibodies to MSP first detect the protein in primary spermatocytes within structures termed fibrous body-membranous organelle (FB-MO) complexes (Figure 1). MSP accumulates in paracrystalline arrays of filaments within the fibrous bodies. These FB-MO complexes segregate into the budding spermatids. Shortly after the spermatids separate from the residual body, the fibrous bodies dissociate and MSP is distributed throughout the cytoplasm. After activation, MSP becomes relocalized to the pseudopod. Ultrastructural analysis (described in greater detail in Section 7) shows a complex network of MSP filaments within the pseudopod. In addition, secretion of MSP serves as a signaling molecule for oocyte maturation and ovulation (Miller et al., 2001); for details of these functions, the reader is directed to excellent reviews (WormBook chapter Control of oocyte meiotic maturation and ovulation; Kim et al., 2013).

\section{Motility and fertilization}

The mechanics of fertilization in the nematode reproductive tract demonstrate the importance of sperm motility in this process. Crawling spermatozoa accumulate in the sperm storage organ known as the spermatheca, which is the site of fertilization. As each oocyte passes through the spermatheca, many spermatozoa are dislodged into the uterus but quickly return to the spermatheca. The reproductive period spans several days, and virtually every spermatozoon is successful at fertilizing an oocyte, so sperm motility must be maintained throughout this interval (Hirsh et al., 1976; Klass et al., 1976; Ward and Carrel, 1979).

Mutational screens for sperm-specific sterility, the Fer or Spe phenotype, have identified a large number of loci necessary for proper sperm function (e.g., see Argon and Ward, 1980; L'Hernault et al., 1988). Mutations in a number of the Spe/Fer genes produce apparently normal spermatids that fail to activate. These immotile cells initially localize to the spermatheca. However, the passage of oocytes rapidly sweeps the mutant sperm into the uterus, where they are unable to return to the spermatheca. Motility is not sufficient to insure functionality, since mutations in several other Spe/Fer genes permit normal activation but not fertilization (e.g., Singson et al., 1998; reviewed in Marcello et al., 2013); however, motility is in all likelihood a necessary prerequisite.

\section{Acquisition of motility}

The maturation of non-motile spermatids into crawling spermatozoa is called activation, or spermiogenesis. Activation occurs differently in the two sexes, reflecting the mechanistic differences of self vs. cross-fertilization (Hirsh et al., 1976; Klass et al., 1976; Ward and Carrel, 1979). In hermaphrodites, gametogenesis switches from 
sperm to oocyte production at the onset of adulthood. Spermatids are propelled ahead of the developing oocytes into the spermatheca, where activation takes place. In males, spermatids are stored in the seminal vesicle until insemination. The ejaculation of spermatids into the hermaphrodite uterus leads to their rapid activation and subsequent movement into the spermatheca.

Activation is mediated by two distinct pathways. Hermaphrodite spermiogenesis requires components of the SPE-8 class, consisting of spe-8, spe-12, spe-19, spe-27, and spe-29, (L'Hernault et al., 1988; Minniti et al., 1996; Nance et al., 2000; Geldziler et al., 2005). Mutations of the SPE-8 class block the activation of spermatids in hermaphrodites but not males. As a consequence, hermaphrodites are self-sterile while males remain cross-fertile. The SPE-8 genes are expressed in sperm, and their predicted molecular functions are consistent with a signal transduction pathway. spe-12, spe-19, and spe-29 encode transmembrane proteins, while spe-8 encodes a putative cytoplasmic tyrosine kinase (Paul Muhlrad and Craig LaMunyon, personal communication). The initiating signal for activation of the SPE-8 pathway is currently unknown.

Evidence for a second, male-specific activation pathway was inferred from the phenomenon of trans-activation. Spermatids from SPE-8 class mutant hermaphrodites can be activated by mating with males, even ones that lack functional sperm (Shakes and Ward, 1989). These trans-activated hermaphrodite sperm become motile and capable of fertilization (further demonstrating the importance of motility for sperm cell function).

Genetic screens for components of the male activation pathway identified try-5, which encodes a serine protease that activates spermiogenesis (Smith and Stanfield, 2011). TRY-5 activity is held in check until insemination by SWM-1, a serine protease inhibitor (Stanfield and Villeneuve, 2006). TRY-5 is a component of male seminal fluid that, in conjunction with SWM-1, mediates the appropriate timing of spermatid activation. The timing is critical for fertility, as precocious activation caused by loss of $s w m-1$ results in male fertility defects. TRY-5 activation is redundant with the SPE-8 program, as male-specific sterility requires inactivation of both pathways. Although the substrate(s) for proteolytic cleavage has not been identified, this mechanism of activation is evolutionarily conserved in the parasitic nematode Ascaris suum (Zhao et al., 2012).

Screens for suppressors of the hermaphrodite sterility caused by the SPE- 8 class of mutations have identified additional components of the signalling pathway: spe-6, which encodes a casein kinase I homolog; spe-4, a presenilin; and spe-46, a seven-pass transmembrane protein (Muhlrad and Ward, 2002; Gosney et al., 2008; Liau et al., 2013). These suppressors can bypass mutations in multiple components of the SPE-8 class, and lead to precocious activation of male spermatids in an otherwise wild-type background. Whereas the suppressors are presumed reduction-of-function mutations, spe- 6 and spe-4 loss-of-function alleles cause sperm-specific sterility in both sexes (L'Hernault and Arduengo, 1992; Varkey et al., 1993). Thus, the SPE-8 and TRY-5 pathways may converge on these downstream components to promote spermatid activation. A summary of the sperm activation pathways is diagrammed in Figure 4.

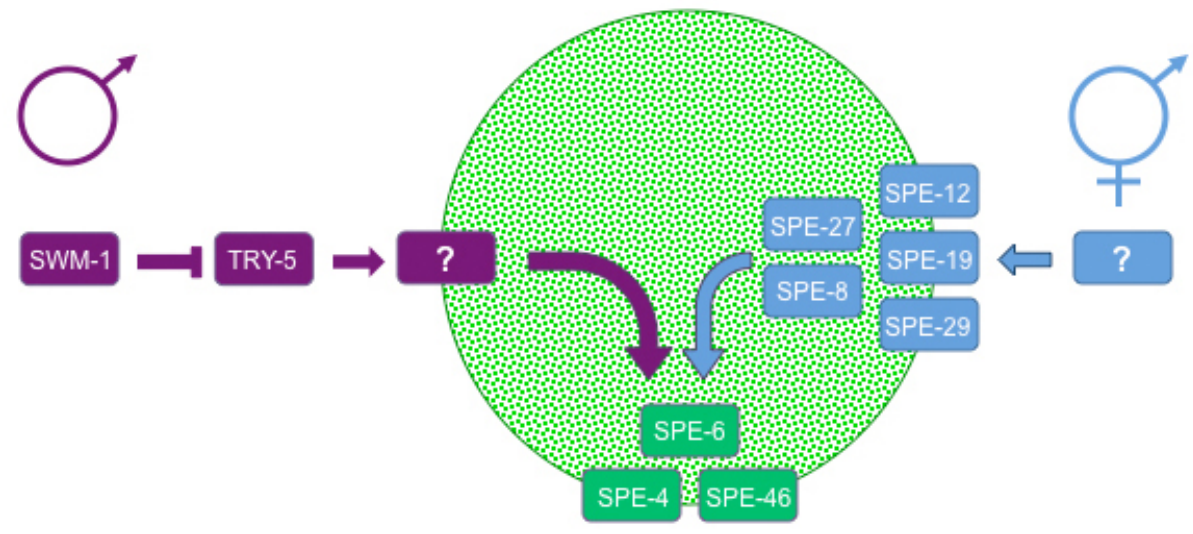

Figure 4. Sperm activation pathway. Genetically defined components of the male (purple) and hermaphrodite (blue) activation pathways. See text for details.

Additional modes of signaling have also been reported. Mutation of cil-1, a phosphoinositide (PI)-5 phosphatase, prevents activation, whereas exposure to the PI 3-kinase inhibitor wortmannin promotes activation of wild-type spermatids (Bae et al., 2009). Similarly, treatment with calmodulin inhibitors such as trifluoperazine can promote spermatid activation (Shakes and Ward, 1989). Currently, it is unclear how either of these signaling 
systems might interact with the SPE-8 and/or TRY-5 pathways. Treatment with zinc is also able to activate spermatids in vitro, and zinc is enriched in the tissues associated with activation in vivo (spermatheca in hermaphrodites, gonadal secretory cells in males). The mechanism of zinc activation is unknown, but it requires components of the SPE-8 class (Liu et al., 2013).

\section{Cytoskeletal restructuring}

Activation occurs in the absence of new protein synthesis, and produces rapid and dynamic rearrangements of cellular morphology (Nelson and Ward, 1980; Shakes and Ward, 1989). One of the earliest hallmarks of activation is the fusion of specialized vesicles known as membranous organelles (MOs) with the plasma membrane. This event coincides with the formation of spiky projections of the cell surface, driven by the assembly of MSP filaments. These spikes coalesce into the pseudopod that provides motility. During activation, different components are partitioned into the cell body and pseudopod, and the two compartments are separated by a series of laminar membranes in mature sperm. Constituents of the cell body include the nucleus, centriole pair, and mitochondria, while the motile apparatus is localized to the pseudopod.

MO fusion during activation appears to be essential for establishing normal cell asymmetry with a fully functional pseudopod. The MOs persist as stable membrane pores that are restricted to the cell body in mature spermatozoa. FER-1 is a membrane component of the MOs that promotes their fusion in a calcium-dependent manner (Washington and Ward, 2006). The failure of MO fusion in fer- 1 mutants is accompanied by the formation of a short pseudopod with limited motility (Argon and Ward, 1980; Achanzar and Ward, 1997). Cholesterol also plays a critical role in spermatid activation and cell asymmetry. Manipulation of cholesterol levels is sufficient to promote or prevent activation, and naturally occuring cholesterol-enriched microdomains become localized to the cell body during pseudopod extension (Dou et al., 2012; Fraire-Zamora et al., 2012).

\section{Motility and MSP}

Video microscopy of crawling sperm (Figure 3) reveals the critical role of MSP polymerization in cell motility (Roberts and Ward, 1982; Sepsenwol et al., 1989). Much of this work is on the large sperm from A. suum, but the same features hold true for $C$. elegans. Movement occurs by extension of the leading edge of the pseudopod, attachment to the substrate, and retraction at the cell body. A meshwork of fibers extends throughout the pseudopod. Assembly of new fibers at the leading edge of the pseudopod and disassembly at its base produce a treadmilling motion, and the rate of treadmilling correlates precisely with the rate of crawling (Roberts and King, 1991). Manipulation of the rates of assembly and disassembly by $\mathrm{pH}$ (see following section) clearly demonstrates that the motive force is coupled to the combination of these two opposing processes (Italiano et al., 1999). In other types of amoeboid cells, motility is based on the actin cytoskeleton. However, nematode spermatozoa contain virtually no actin (Nelson et al., 1982), but instead employ MSP filaments in this role.

\section{6. $\mathrm{pH}$ regulation}

Intact spermatids can be obtained from males in bulk, and a variety of agents are able to promote activation in vitro. These experiments reveal a critical role for $\mathrm{pH}$ in regulating spermiogenesis. Agents that increase the intracellular $\mathrm{pH}$ (e.g., the weak base triethanolamine or the ionophore monensin at basic $\mathrm{pH}$ ) induce spermatid activation (Ward et al., 1983). The molecular mechanism of this activation is unknown; however, it is likely physiologically relevant, since in vitro activation by triethanolamine produces mature spermatozoa capable of oocyte fertilization upon artificial insemination (LaMunyon and Ward, 1994). Indeed, initiation of the spermiogenesis pathway might culminate in elevated $\mathrm{pH}$, since activation by triethanolamine or monensin bypasses the requirement for the SPE-8 class signaling components (Shakes and Ward, 1989).

Changes in intracellular $\mathrm{pH}$ appear to control not just activation but also MSP polymerization throughout spermatogenesis (King et al., 1994a). Experiments in A. suum show that intracellular pH is highest in spermatocytes, when MSP is assembled into paracrystalline arrays in fibrous bodies, and lowest in spermatids, when MSP disassembles into the cytosol. Spermiogenesis induces an increase in $\mathrm{pH}$ and the assembly of MSP macrofibers. Remarkably, a pH gradient forms within the pseudopod of the spermatozoon; a higher $\mathrm{pH}$ is observed at the leading edge, where new filaments assemble, than at the base, where disassembly occurs. Decreasing the $\mathrm{pH}$ by buffer treatment can either slow or halt MSP polymerization at the leading edge of the pseudopod without affecting disassembly. A return to physiological buffer rapidly restores MSP assembly, pseudopod extension, and motility (Italiano et al., 1999). Thus, MSP assembly in all cases is accompanied by an increase in intracellular pH. In vitro filament assembly occurs across a wide range of $\mathrm{pH}$, so direct regulation of MSP polymerization by $\mathrm{pH}$ is unlikely (King et al., 1992); rather, $\mathrm{pH}$ appears to alter the activity of components that control the assembly of MSP filaments. 


\section{MSP assembly and structure}

MSP polymerization occurs through a series of increasingly higher-order interactions (King et al., 1994b). Pairs of MSP monomers produce dimers, dimers assemble into helical subfilaments, pairs of subfilaments entwine to form helical filaments, and filaments associate into larger structures called fibers, macrofibers, or bundles. MSP dimers are extremely stable in solution $\left(\mathrm{K}_{\mathrm{d}}<5 \times 10^{-8}\right)$ and exhibit the same structure as in the filamentous form (Haaf et al., 1996); therefore, the polymerization of MSP is likely regulated at the level of subfilament assembly.

Structural studies have provided insights into the mechanics of MSP assembly that generate the motive force. X-ray diffractions of MSP crystals from both A. suum (Bullock et al., 1996) and C. elegans (Baker et al., 2002) reveal an immunoglobulin-like fold comprised of a seven-stranded $\beta$ sandwich (Figure 5A). MSP forms symmetrical dimers through $\beta$ strands $a$ and $b$ plus the penultimate asparagine residue (Figure 5B). The assembly of dimers into subfilaments occurs through hydrogen bonding between residues within strand $g$ (Figure 5C). Filament formation between subfilaments is mediated through multiple sites of interaction, and the same sites might function in filament bundling as well. Mutational and biochemical analyses of recombinant MSP have confirmed the importance of residues critical for the immunoglobulin fold, the dimerization interface, and subfilament assembly (Smith and Ward, 1998; del Castillo-Olivares and Smith, 2008).
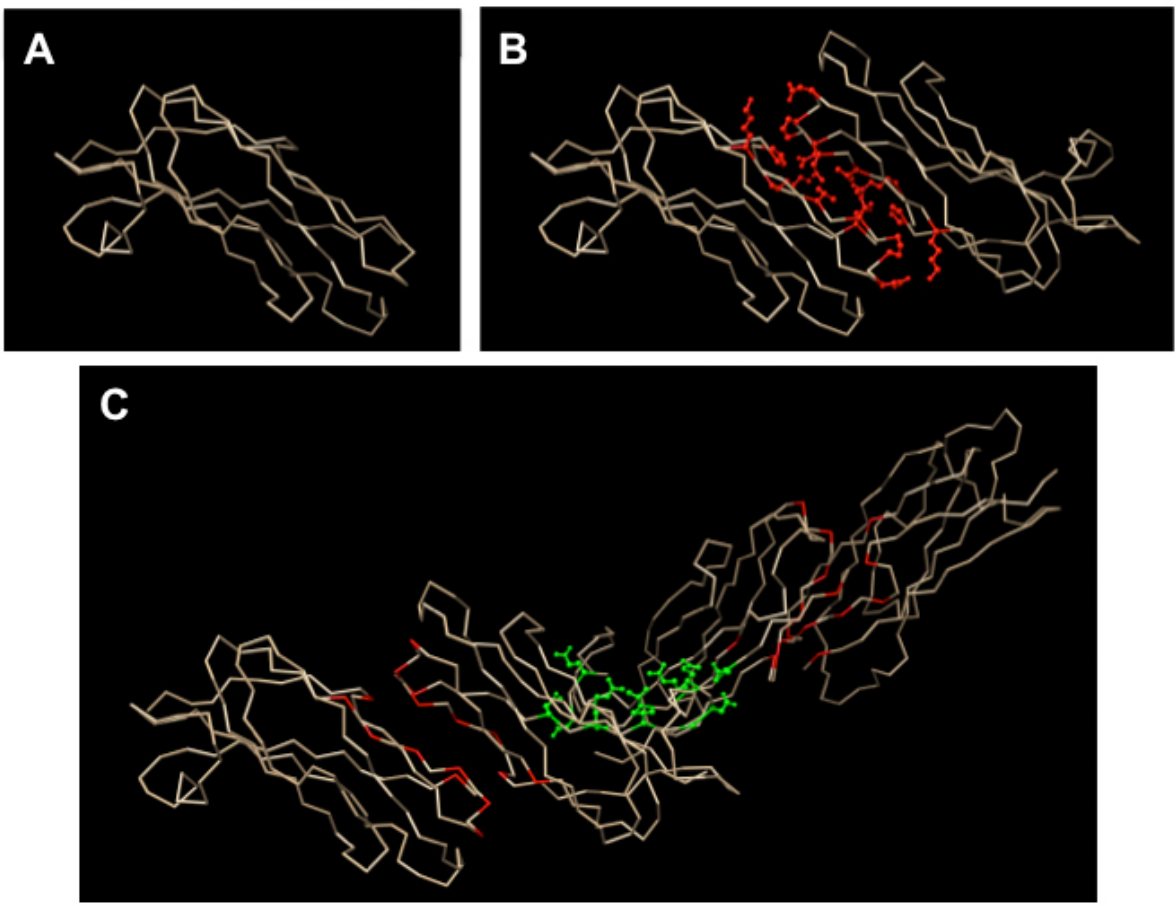

Figure 5. Structure of . elegans MSP. (A) MSP monomer. Backbone trace demonstrates the seven-stranded immunoglobulin-like fold. (B) MSP dimer. Sidechains involved in dimer formation are shown in red. (C) MSP subfilament. Sidechains involved in subfilament interaction between two dimers are shown in green. The leftmost monomer in B and C is the same size and orientation as in A. Structure coordinates are available as entry 1GRW from the Protein Data Bank.

Purified MSP exists as a dimer under most buffer conditions; however, polymerization occurs in the presence of polyethylene glycol or water-miscible alcohols (King et al., 1992). Transmission electron micrographs of these negatively stained filaments are indistinguishable from those obtained from fixed spermatozoa, suggesting that filament formation is an intrinsic property of MSP even in the absence of additional proteins.

\section{Reconstituted MSP polymerization system}

A crucial advance in our understanding of MSP-based motility has been permitted by the development of a reconstituted cell-free filament assembly system (Italiano et al., 1996). Addition of ATP to lysate from A. suum sperm causes the assembly of MSP into fibers. Fractionation of the lysate demonstrates that both membrane and cytosolic components, in addition to MSP and ATP, are necessary for fiber formation. Phase-contrast video microscopy reveals that each MSP fiber is nucleated by a membrane vesicle, and that the vesicle is propelled 
forward by the growing fiber. Furthermore, only the subset of vesicles that are derived from the leading edge of the pseudopod, the site of MSP polymerization in vivo, can promote MSP filament assembly in vitro. The maximum observed rate of fiber growth is similar to the rate of sperm crawling, suggesting that all of the relevant components are present.

The disassembly of MSP fibers can be promoted in the same cell-free system (Miao et al., 2003). Following fiber growth from reconstituted components, removal of ATP and addition of a tyrosine phosphatase to sperm extracts causes fiber retraction. A bead attached to the non-vesicle end of the MSP fiber is drawn toward the vesicle end as the fiber shortens. The phosphatase controls the activity of a disassembly factor, since MSP itself does not appear to be phosphorylated (although see Fraire-Zamora et al., 2011). Thus, the reconstituted system exhibits the primary hallmarks of in vivo motility: the assembly of MSP fibers at the leading edge of the pseudopod membrane generates a motive force that propels the membrane forward, and disassembly causes fiber retraction at the trailing edge.

\section{Components that control MSP assembly}

Investigators have used the reconstituted system from $A$. suum to identify proteins that regulate the assembly of MSP. One critical component from the vesicle fraction is a $48 \mathrm{kDa}$ integral membrane protein with the unwieldy name major sperm protein polymerization organizing protein or, more simply, MPOP (LeClaire et al., 2003). Phosphorylation of tyrosine residues appears to regulate the activity of MPOP. Whereas the protein is evenly distributed throughout the sperm plasma membrane, anti-phosphotyrosine labeling of the membrane is restricted to the leading edge of the pseudopod. Purified phosphorylated MPOP, when combined with cytosol and ATP, is sufficient to promote MSP filament assembly in the absence of other vesicle components. Intriguingly, lowering the $\mathrm{pH}$ blocks both tyrosine phosphorylation of MPOP and the in vivo assembly of MSP in a similar manner. This observation suggests an appealing mechanism for the regulation of motility by $\mathrm{pH}$, whereby an as-yet-unidentified, $\mathrm{pH}$-sensitive tyrosine kinase phosphorylates MPOP at the tip of the pseudopod to nucleate the polymerization of MSP fibers.

A primary function of MPOP is the recruitment of MSP polymerization activating kinase (MPAK) to the site of MSP assembly. MPAK is a cytosolic serine/threonine kinase of the casein kinase I family that co-localizes with phosphorylated MPOP in vivo and in vitro, and its activity is required for MSP fiber formation in the reconstituted system (Yi et al., 2007). MPAK phosphorylates a structural component of the MSP cytoskeleton called MFP2 (for MSP fiber protein). MFP2 appears to be essential for fiber formation (Buttery et al., 2003). Anti-MFP2 antibodies abrogate MSP filament assembly in the reconstituted system, and addition of purified MFP2 stimulates fiber growth. Phospho-MFP2 is incorporated into MSP fibers, and its crystal structure combined with its role in promoting polymerization suggests a role analogous to formins in actin assembly (Grant et al., 2005).

A family of small (15-16 kDa) cytosolic proteins plays the opposite role in MSP assembly, as a negative regulator of fiber growth. Members include MFP1 $\alpha, \beta$, and $\gamma$, which were identified biochemically as components of MSP fibers (Buttery et al., 2003). Subsequently, MDP-1 and MDP-6 were identified by molecular methods (Tarr and Scott, 2005a; Tarr and Scott, 2005b). Anti-MFP1 antibodies promote fiber formation in the reconstituted system, while addition of purified MFP1 inhibits MSP polymerization. Homologs in C. elegans comprise a larger gene family of sperm-specific proteins. The crystal structure of one member, encoded by $s s p-19$, is remarkably similar to MSP despite having less than 20\% amino acid sequence identity (Schormann et al., 2004). This structural similarity implies a role as either a capping protein or a competitive inhibitor of MSP polymerization.

MSP disassembly and fiber retraction is mediated by another cytoskeletal protein called MFP3. Threonine-phosphorylated MFP3 is a stabilizing component of MSP filaments (Yi et al, 2009). Dephosphorylation of MFP3 causes dissociation from the fibers and their subsequent disassembly. Protein phosphatase 2A (PP2A) is responsible for MFP3 dephosphorylation in vitro, and its activity in turn is promoted by tyrosine dephosphorylation. PP2A from sperm extracts is phosphorylated; the promotion of fiber retraction in the reconstituted system by addition of exogenous tyrosine phosphatase presumably reflects the activation of PP2A (Miao et al., 2003). In vivo, PP2A is concentrated at the base of the pseudopod, the site of MSP depolymerization and retraction (Yi et al, 2009). The endogenous tyrosine kinase and phosphatase that regulate PP2A are currently unidentified. A summary of the factors that regulate MSP polymerization is diagrammed in Figure 6. 


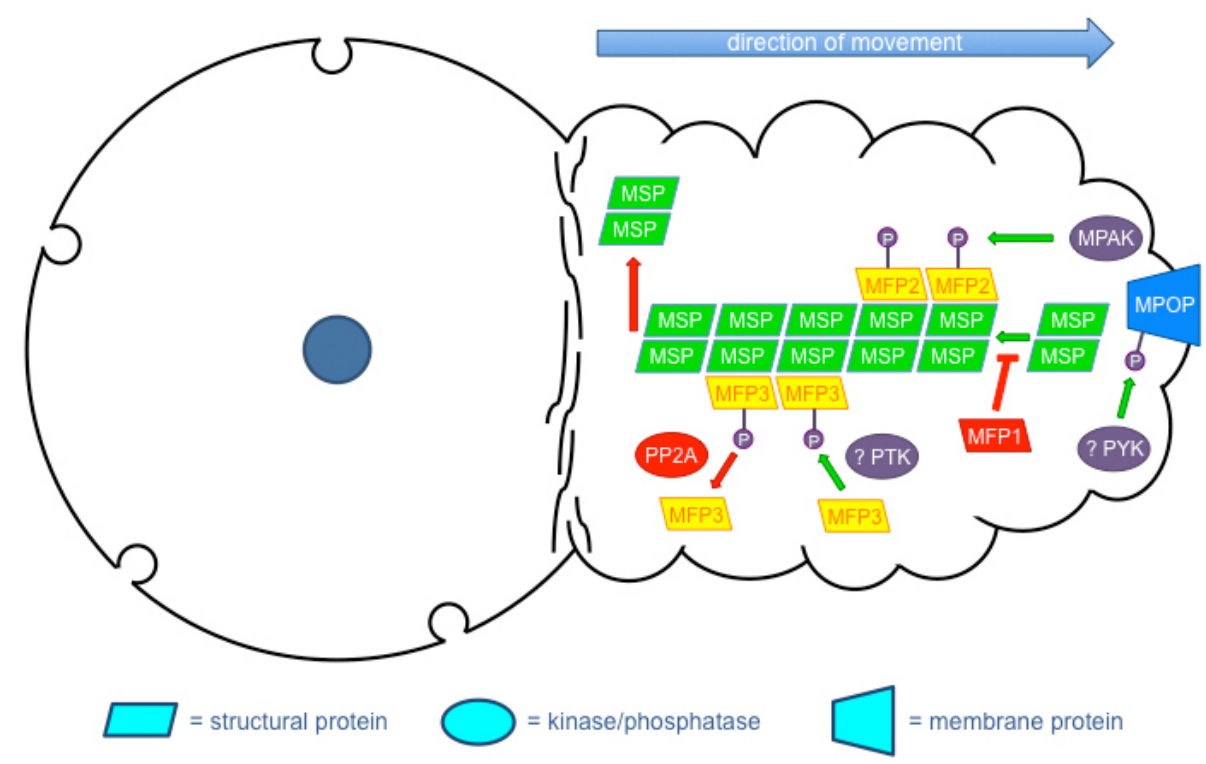

Figure 6. Regulation of MSP assembly. Components identified by reconstituted MSP polymerization assay. Polymerization-enhancing steps indicated by green arrows; depolymerization steps in red. Phosphorylation of MPOP by an unidentified protein tyrosine kinase (PYK) at the tip of the pseudopod promotes MPAK recruitment. MPAK phosphorylates MFP2, which stimulates MSP assembly, while MFP1 antagonizes MSP assembly. Phosphorylation of MFP3 by an unidentified protein threonine kinase (PTK) stabilizes MSP filaments. Dephosphorylation of MFP3 by PP2A at the base of the pseudopod destabilizes the filament and drives disassembly of MSP.

Although the proteins that direct MSP assembly in C. elegans are largely unknown, sequence conservation suggests that the same components are likely to be involved. Homologs of all components excepting MPOP (whose complete sequence has not been determined) are present in C. elegans, and microarray analyses indicate that these genes are expressed in the germline during spermatogenesis (Reinke et al., 2000; Reinke et al., 2004). Furthermore, a functional equivalence is found between PP2A in A. suum and the PP1-class protein phosphatases GSP-3/4 in $C$. elegans. Mutations in $g s p-3 / 4$ cause sperm-specific sterility due to defects in motility, and localization of GSP-3/4 at the base of the pseudopod strongly implies a role in MSP disassembly analogous to PP2A (Wu et al., 2012).

\section{Directionality and force generation}

Filamentous actin and microtubules possess an inherent orientation due to the asymmetry of their constituent molecules. However, MSP filaments are composed of symmetrical dimers that, like train tracks, lack an intrinsic polarity. The directionality of filament assembly and disassembly must be imposed externally by the asymmetric distribution of the proteins responsible for these processes. Phosphorylated MPOP is restricted to the leading edge of the pseudopod membrane, where it directs MSP assembly via MPAK recruitment/MFP2 phosphorylation. At the base of the pseudopod, the localization of active PP2A dephosphorylates MFP3, which promotes MSP depolymerization. This distribution of components ensures that MSP assembly drives protrusion at the tip of the pseudopod, while disassembly at the base pulls the cell body forward.

Components common to other examples of cell motility-actin, motor proteins, and microtubules (with the exception of the centrioles) - segregate into the residual body and are excluded from the spermatid. Therefore, the motive force in nematode spermatozoa appears to be driven solely by the assembly of MSP. Propulsion by polymerization poses a physical challenge, since the filament end must abut the plasma membrane to generate protrusion while simultaneously being accessible for growth by subunit addition. One might predict that MSP assembly and sperm motility would be opposed by membrane tension, but the opposite phenomenon is observed. The degree of membrane tension correlates positively with the rate of cell motility, which reflects an increase in both the length of MSP fibers and their orientation relative to the direction of movement (Batchelder et al., 2011). Cell speed increases with elongation of the pseudopod (Zajac et al., 2008), which would also increase membrane tension. Mathematical modeling of this relationship between speed and elongation requires elastic anisotropy, i.e., increased stiffness of the cytoskeleton in the direction of movement.

The standard theoretical model for polymerization-based motility is an 'elastic Brownian ratchet', in which thermal fluctuations of the flexible filament provide transient access for the incorporation of new subunits (Mogilner and Oster, 1996). However, ultrastructural studies of MSP fibers reveal a previously unappreciated contribution of 
filament-packing dynamics to the generation of force (Miao et al., 2008). The region immediately contacting the membrane contains a high-density meshwork of short, elongating fibers. Fiber length increases with distance from the membrane, but these longer fibers cannot pack as densely as shorter fibers. Decreased fiber-packing density produces a concomitant increase in the volume of the fiber meshwork, which generates force through the elongating fibers to produce membrane expansion. Similarly, filament packing contributes to force generation during retraction (Shimabukuro et al., 2011). As some of the filaments undergo depolymerization at the base of the fiber, the remaining filaments repack into a smaller volume. This contraction of the fiber meshwork pulls the base forward (Figure 7).

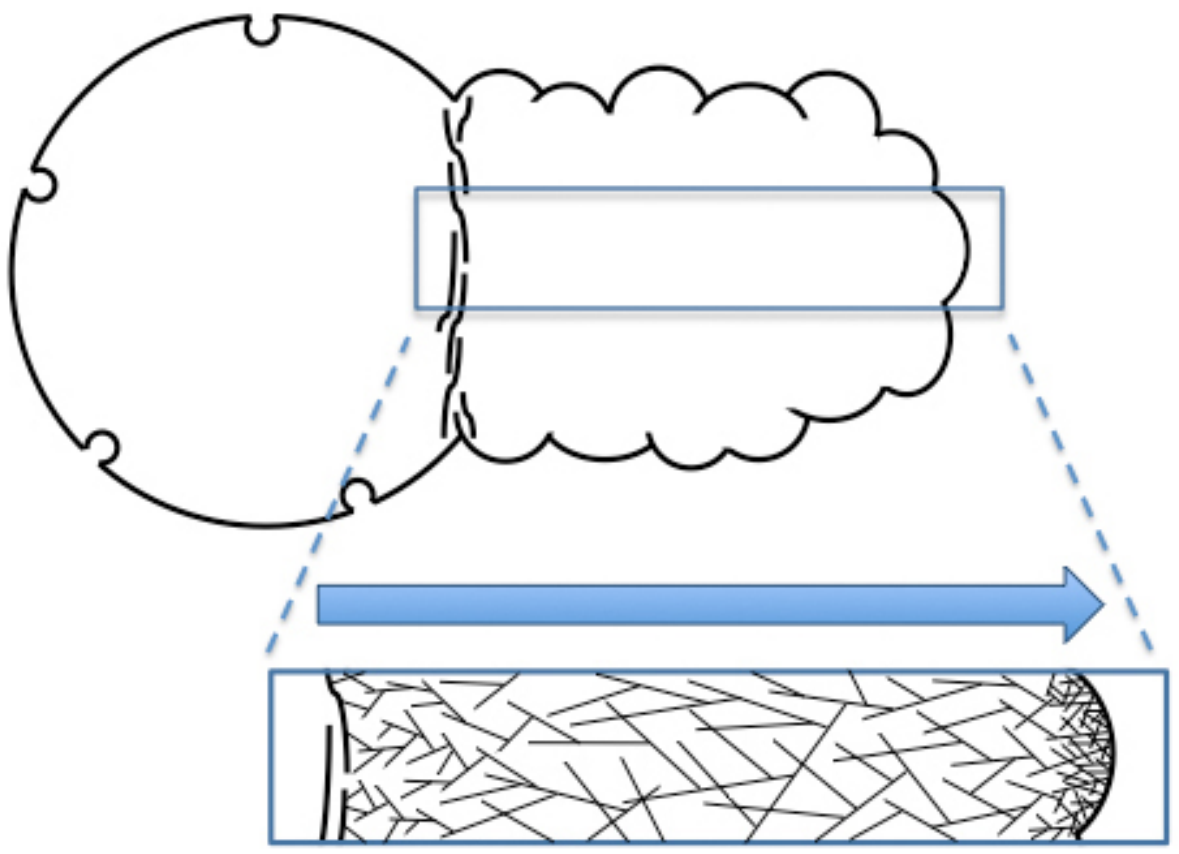

Figure 7. Force generation by filament packing. Schematic diagram of MSP filament dynamics. Nucleation of MSP assembly at the leading edge of the pseudopod (head of arrow) produces numerous short, densely packed filaments. Increasing filament length behind the leading edge reduces packing density, thereby increasing filament volume and generating force. MSP disassembly at the trailing edge of the pseudopod (tail of arrow) allows filament repacking at higher density, which decreases volume and propels the cell body forward.

\section{Guidance cues}

Spermatozoa relocalize efficiently to the spermatheca after displacement by passing oocytes, suggesting the presence of an attractant cue. Similarly, male spermatozoa migrate rapidly from the vulva to the spermatheca after insemination. Tracking assays with fluorescently labeled male sperm indicate that oocytes are the source of an attractant(s), and that the signal is derived from polyunsaturated fatty acids, or PUFAs (Kubagawa et al., 2006). Mutations that disrupt PUFA synthesis (fat-2 and fat-3) or PUFA import into oocytes ( rme-2) cause sperm migration defects with reduced brood sizes, which can be rescued by dietary supplementation of PUFAs. Subsequent analysis identified a mixture of F-series prostaglandins synthesized from PUFAs in the oocytes as an attractant for sperm guidance (Hoang et al., 2013). Additional components of this pathway include the FOXO transcription factor DAF-16, which regulates targets necessary for PUFA import into oocytes (Edmonds et al., 2010), and the gap junction protein INX-14, which functions downstream of prostaglandin synthesis (Whitten and Miller, 2007; Edmonds et al., 2011).

\section{Evolution of the MSP gene family}

MSP-based sperm motility appears to be conserved among nematodes. Crawling spermatozoa are rare among metazoans, but they are a characteristic feature of this phylum. In C. elegans, MSP is encoded by a family of 28 genes (not including several additional pseudogenes) that produce isoforms that are 97-100\% identical (Burke and Ward, 1983; Klass et al., 1984; Ward et al., 1988). MSP genes have been identified across widely diverged nematode species (e.g., see Scott et al., 1989) and MSP gene expression is restricted to the sperm (Klass et al., 1982; Ward, 1987). MSPs from all nematodes are greater than $60 \%$ identical, with particularly high sequence conservation in the dimerization interface as well as $\beta$ strands $f$ and $g$ (the proposed site of subfilament assembly). Mutational 
studies indicate a functional conservation. Missense mutations that abolish MSP-MSP interaction in the yeast two-hybrid system map almost exclusively to the conserved regions (Smith and Ward, 1998), and these mutations also disrupt MSP filament assembly in vitro (del Castillo-Olivares and Smith, 2008). However, conservation might reflect the role(s) of MSP in oocyte maturation and/or ovulation signaling rather than motility (Miller et al., 2001).

MSP seems to be restricted to nematodes, so how did this unique mechanism of cell motility arise? Although homologs have not been found in other phyla, proteins with limited sequence similarity have been identified in species from plants to mammals. The first of these, VAP-33, was identified from Aplysia californica as a VAMP/synaptobrevin-interacting protein involved in neurotransmitter release from synaptic vesicles (Skehel et al., 1995). Subsequent studies in other organisms indicate a more general role for VAP proteins in vesicle fusion, trafficking, and cell signaling (Skehel et al., 2000). All of the VAP homologs are predicted to share a similar architecture: an amino-terminal MSP-like domain, a central coiled-coil region, and a carboxy-terminal transmembrane domain (Laurent et al., 2000). VAP homologs with the same structure are also present in C. elegans. Thus, it appears that the small, cytosolic MSP arose from a larger, membrane-tethered protein.

Likewise, the function of MSP as a cytoskeletal protein underlying sperm cell motility is undoubtedly a derived property. The human VAPB/ALS8 gene encodes a VAP whose MSP-like domain is cleaved and secreted, where it functions as an extracellular ligand for Eph receptors (Tsuda et al., 2008). An identical role is observed for MSP in C. elegans: the protein is secreted from sperm and binds to the VAB-1 Eph receptor to promote oocyte maturation (Miller et al., 2003). This activity of the MSP domain as a ligand for Eph receptors likely reflects its ancestral function.

\section{Conclusion}

Despite the apparent novelty of MSP and sperm motility, the system can offer insights into canonical, actin-based mechanisms of cell movement. The generation of force and membrane protrusion by protein polymerization is a common feature of both systems. Actin is required for multiple cellular processes, which greatly complicates the analysis of its role in motility. In contrast, the relative simplicity of sperm motility offers an attractive model for investigation, and the combination of genetic and biochemical approaches will continue to advance our understanding of the underlying biomechanical properties of amoeboid cell movement.

\section{References}

Achanzar, W.E., and Ward, S. (1997). A nematode gene required for sperm vesicle fusion. J. Cell Sci. 110, 1073-1081. Abstract

Argon, Y., and Ward, S. (1980). Caenorhabditis elegans fertilization-defective mutants with abnormal sperm. Genetics 96, 413-433. Abstract

Bae, Y.K., Kim, W., L'Hernault, S.W., and Barr, M.M. (2009). The CIL-1 PI 5-phosphatase localizes TRP polycystins to cilia and activates sperm in C. elegans. Curr. Biol. 19, 1599-1607. Abstract Article

Baker, A.M., Roberts, T.M., and Stewart, M. (2002). $2.6 \AA$ resolution crystal structure of helices of the motile major sperm protein (MSP) of Caenorhabditis elegans. J. Mol. Biol. 319, 491-499. Abstract Article

Batchelder, E.L., Hollopeter, G., Campillo, C., Mezanges, X., Jorgensen, E.M., Nassoy, P., Sens, P., and Plastino, J. (2011). Membrane tension regulates motility by controlling lamellipodium organization. Proc. Natl. Acad. Sci. U. S. A. 108, 11429-11434. Abstract Article

Bullock, T.L., Roberts, T.M., and Stewart, M. (1996). $2.5 \AA$ resolution crystal structure of the motile major sperm protein (MSP) of Ascaris suum. J. Mol. Biol. 263, 284-296. Abstract Article

Burke, D.J., and Ward, S. (1983). Identification of a large multigene family encoding the major sperm protein of Caenorhabditis elegans. J. Mol. Biol. 171, 1-29. Abstract Article

Buttery, S.M., Ekman, G.C., Seavy, M., Stewart, M., and Roberts, T.M. (2003). Dissection of the Ascaris sperm motility machinery identifies key proteins involved in major sperm protein-based amoeboid locomotion. Mol. Biol. Cell 14, 5082-5088. Abstract Article 
Chu, D.S., and Shakes, D.C. (2013). Spermatogenesis. Adv. Exp. Med. Biol. 757, 171-203. Abstract

del Castillo-Olivares, A., and Smith, H.E. (2008). Critical contact residues that mediate polymerization of nematode major sperm protein. J. Cell. Biochem. 104, 477-487. Abstract Article

Dou, J., Chen, L., Hu, Y., and Miao, L. (2012). Cholesterol and the biosynthesis of glycosphingolipids are required for sperm activation in Caenorhabditis elegans. Biochim. Biophys. Acta 1821, 934-942. Abstract Article

Edmonds, J.W., McKinney, S.L., Prasain, J.K., and Miller, M.A. (2011). The gap junctional protein INX-14 functions in oocyte precursors to promote C. elegans sperm guidance. Dev. Biol. 359, 47-58. Abstract Article

Edmonds, J.W., Prasain, J.K., Dorand, D., Yang, Y., Hoang, H.D., Vibbert, J., Kubagawa, H.M., and Miller, M.A. (2010). Insulin/FOXO signaling regulates ovarian prostaglandins critical for reproduction. Dev. Cell 19, 858-871. Abstract Article

Friare-Zamora, J.J., Broitman-Maduro, G., Maduro, M., and Cardullo, R.A. (2011). Evidence for phosphorylation in the MSP cytoskeletal filaments of amoeboid spermatozoa. Int. J. Biochem. Mol. Biol. 2, 263-273. Abstract

Fraire-Zamora, J.J., Tran T., and Cardullo, R.A. (2012). Cholesterol-enriched microdomains regulate pseudopod extension in the MSP-based cytoskeleton of amoeboid sperm. Biochem. Biophys. Res. Comun. 427, 478-484. Abstract Article

Geldziler, B., Chatterjee, I., and Singson, A. (2005). The genetic and molecular analysis of spe-19, a gene required for sperm activation in Caenorhabditis elegans. Dev. Biol. 283, 424-436. Abstract Article

Gosney, R., Liau, W.S., and LaMunyon, C.W. (2008). A novel function for the presenilin family member spe-4: inhibition of spermatid activation in Caenorhabditis elegans. BMC Dev. Biol. 8, 44. Abstract

Grant, R.P., Buttery, S.M., Ekman, G.C. Roberts, T.M., and Stewart, M. (2005). Structure of MFP2 and its function in enhancing MSP polymerization in Ascaris sperm amoeboid motility. J. Mol. Biol. 347, 583-595. Abstract Article

Greenstein, D. Control of oocyte meiotic maturation and fertilization (December 28, 2005), WormBook, ed. The $C$. elegans Research Community, WormBook, doi/10.1895/wormbook.1.53.1, http://www.wormbook.org.

Haaf, A., Butler, P.J., Kent, H.M., Fearnley, I.M., Roberts, T.M., Neuhaus, D., and Stewart, M. (1996). The motile major sperm protein (MSP) from Ascaris suum is a symmetric dimer in solution. J. Mol. Biol. 260, 251-260. Abstract Article

Hirsh, D., Oppenheim, D., and Klass, M. (1976). Development of the reproductive system of Caenorhabditis elegans. Dev. Biol. 49, 200-219. Abstract Article

Hoang, H.D., Prasain, J.K., Dorand, D., and Miller, M.A. (2013). A heterogeneous mixture of F-series prostaglandins promotes sperm guidance in the Caenorhabditis elegans reproductive tract. PLoS Genet. 9, e1003271. Abstract Article

Italiano, J.E., Jr., Roberts, T.M., Stewart, M., and Fontana, C.A. (1996). Reconstitution in vitro of the motile apparatus from the amoeboid sperm of Ascaris shows that filament assembly and bundling move membranes. Cell 84, 105-114. Abstract Article

Italiano, J.E., Jr., Stewart, M., and Roberts, T.M. (1999). Localized depolymerization of the major sperm protein cytoskeleton correlates with the forward movement of the cell body in the amoeboid movement of nematode sperm. J. Cell Biol. 146, 1087-1096. Abstract Article

Kim, S., Spike, C., Greenstein, D. (2013). Control of oocyte growth and meiotic maturation in Caenorhabditis elegans. Adv. Exp. Med. Biol. 757, 277-320. Abstract

King, K.L., Essig, J., Roberts, T.M., and Moerland, T.S. (1994a). Regulation of the Ascaris major sperm protein (MSP) cytoskeleton by intracellular pH. Cell Motil. Cytoskeleton 27, 193-205. Abstract Article 
King, K.L., Stewart, M., and Roberts, T.M. (1994b). Supramolecular assemblies of the Ascaris suum major sperm protein (MSP) associated with amoeboid cell motility. J. Cell Sci. 107, 2141-2149. Abstract

King, K.L., Stewart, M., Roberts, T.M., and Seavy, M. (1992). Structure and macromolecular assembly of two isoforms of the major sperm protein (MSP) from the amoeboid sperm of the nematode, Ascaris suum. J. Cell Sci. 101, 847-857. Abstract

Klass, M.R., Dow, B., and Herndon, M. (1982). Cell-specific transcriptional regulation of the major sperm protein in Caenorhabditis elegans. Dev. Biol. 93, 152-164. Abstract Article

Klass, M.R., Kinsley, S., and Lopez, L.C. (1984). Isolation and characterization of a sperm-specific gene family in the nematode Caenorhabditis elegans. Mol. Cell. Biol. 4, 529-537. Abstract Article

Klass, M., Wolf, N., and Hirsh, D. (1976). Development of the male reproductive system and sexual transformation in the nematode Caenorhabditis elegans. Dev. Biol. 52, 1-18. Abstract Article

Kubagawa, H.M., Watts, J.L., Corrigan, C., Edmonds, J.W., Sztul, E., Browse, J., and Miller, M.A. (2006). Oocyte signals derived from polyunsaturated fatty acids control sperm recruitment in vivo. Nat. Cell Biol. 8, 1143-1148. Abstract Article

LaMunyon, C.W., and Ward, S. (1994). Assessing the viability of mutant and manipulated sperm by artificial insemination of Caenorhabditis elegans. Genetics 138, 689-692. Abstract

Laurent, F., Labesse, G., and de Wit, P. (2000). Molecular cloning and partial characterization of a plant VAP33 homologue with a major sperm protein domain. Biochem. Biophys. Res. Commun. 270, 286-292. Abstract Article

LeClaire, L.L. III, Stewart, M., and Roberts, T.M. (2003). A 48 kDa integral membrane phosphoprotein orchestrates the cytoskeletal dynamics that generate amoeboid cell motility in Ascaris sperm. J. Cell Sci. 116, 2655-2663. Abstract Article

L'Hernault, S.W. Spermatogenesis (February 20, 2006), WormBook, ed. The C. elegans Research Community, WormBook, doi/10.1895/wormbook.1.85.1, http://www.wormbook.org.

L'Hernault, S.W., and Arduengo, P.M. (1992). Mutation of a putative membrane protein in Caenorhabditis elegans prevents sperm differentiation but not its associated meiotic divisions. J. Cell Biol. 119, 55-68. Abstract Article

L'Hernault, S.W., Shakes, D.C., and Ward, S. (1988). Developmental genetics of chromosome I spermatogenesis-defective mutants in the nematode Caenorhabditis elegans. Genetics 120, 435-452. Abstract

Liau, W.S., Nasri, U., Elmatari, D., Rothman, J., and LaMunyon, C.W. (2013). Premature sperm activation and defective spermatogenesis caused by loss of spe-46 function in Caenorhabditis elegans. PLoS One 8, e57266. Abstract Article

Liu, Z., Chen, L., Shang, Y., Huang, P., and Miao, L. (2013). The micronutrient element zinc modulates sperm activation through the SPE-8 pathway in Caenorhabditis elegans. Development 140, 2103-2107. Abstract Article

Marcello, M.R., Singaravelu, G., and Singson, A. (2013). Fertilization. Adv. Exp. Med. Biol. 757, 321-350. Abstract

Miao, L., Vanderlinde, O., Stewart, M., and Roberts, T.M. (2003). Retraction in amoeboid cell motility powered by cytoskeletal dynamics. Science 302, 1405-1407. Abstract Article

Miao, L., Vanderlinde, O., Liu, J., Grant, R.P., Wouterse, A., Shimabukuro, K., Philipse, A., Stewart, M., and Roberts, T.M. (2008). The role of filament-packing dynamics in powering amoeboid cell motility. Proc. Natl. Acad. Sci. U. S. A. 105, 5390-5395. Abstract Article

Miller, M.A., Nguyen, V.Q., Lee, M.H., Kosinski, M., Schedl, T., Caprioli, R.M., and Greenstein, D. (2001). A sperm cytoskeletal protein that signals oocyte meiotic maturation and ovulation. Science 291, 2144-2147. Abstract Article 
Miller, M.A., Ruest, P.J., Kosinski, M., Hanks, S.K., and Greenstein, D. (2003). An Eph receptor sperm-sensing control mechanism for oocyte meiotic maturation in Caenorhabditis elegans. Genes Dev. 17, 187-200. Abstract Article

Minniti, A.N., Sadler, C., and Ward, S. (1996). Genetic and molecular analysis of spe-27, a gene required for spermiogenesis in Caenorhabditis elegans hermaphrodites. Genetics 143, 213-223. Abstract Article

Mogilner, A., and Oster, G. (1996). Cell motility driven by actin polymerization. Biophys. J. 71, 3030-3045. Abstract Article

Muhlrad, P.J., and Ward, S. (2002). Spermiogenesis initiation in Caenorhabditis elegans involves a casein kinase I encoded by the spe-6 gene. Genetics 161, 143-155. Abstract

Nance, J., Davis, E.B., and Ward, S. (2000). spe-29 encodes a small predicted membrane protein required for the initiation of sperm activation in Caenorhabditis elegans. Genetics 156, 1623-1633. Abstract

Nelson, G.A., Roberts, T.M., and Ward, S. (1982). Caenorhabditis elegans spermatozoan locomotion: amoeboid movement with almost no actin. J. Cell Biol. 92, 121-131. Abstract

Nelson, G.A., and Ward, S. (1980). Vesicle fusion, pseudopod extension and amoeboid motility are induce in nematode spermatids byt the ionophore monensin. Cell 19, 457-464. Abstract Article

Reinke, V., Smith, H.E., Nance, J., Wang, J., Van Doren, C., Begley, R., Jones, S.J., Davis, E.B., Scherer, S., Ward, et al. (2000). A global profile of germline gene expression in C. elegans. Mol. Cell 6, 605-616. Abstract Article

Reinke, V., Gil, I.S., Ward, S., and Kazmer, K. (2004). Genome-wide germline-enriched and sex-biased expression profiles in Caenorhabditis elegans. Development 131,311-323. Abstract Article

Roberts, T.M., and King, K.L. (1991). Centripetal flow and directed reassembly of the major sperm protein (MSP) cytoskeleton in the amoeboid sperm of the nematode, Ascaris suum. Cell Motil. Cytoskeleton 20, 228-241. Abstract Article

Roberts, T.M., Pavalko, F.M., and Ward, S. (1986). Membrane and cytoplasmic proteins are transported in the same organelle complex during nematode spermatogenesis. J. Cell Biol. 102, 1787-1796. Abstract

Roberts, T.M., and Ward, S. (1982). Centripetal flow of pseudopodial surface components could propel the amoeboid movement of Caenorhabditis elegans spermatozoa. J. Cell Biol. 92, 132-138. Abstract

Schormann, N., Symersky, J., and Luo, M. (2004). Structure of sperm-specific protein SSP-19 from Caenorhabditis elegans. Acta Crystallogr. D Biol. Crystallogr. 60, 1840-1845. Abstract Article

Scott, A.L., Dinman, J., Sussman, D.J., and Ward, S. (1989). Major sperm protein and actin genes in free-living and parasitic nematodes. Parisitology 98, 471-478. Abstract Article

Sepsenwol, S., Ris, H., and Roberts, T.M. (1989). A unique cytoskeleton associated with crawling in the amoeboid sperm of the nematode, Ascaris suum. J. Cell Biol. 108, 55-66. Abstract

Shakes, D.C., and Ward, S. (1989). Initiation of spermiogenesis in C. elegans: a pharmacological and genetic analysis. Dev. Biol. 134, 189-200. Abstract Article

Shimabukuro, K., Noda, N., Stewart, M., and Roberts, T.M. (2011). Reconstitution of amoeboid motility in vitro identifies a motor-independent mechanism for cell body retraction. Curr. Biol. 21, 1727-1731. Abstract Article

Singson, A., Mercer, K.B., and L'Hernault, S.W. (1998). The C. elegans spe-9 gene encodes a sperm transmembrane protein that contains EGF-like repeats and is required for fertilization. Cell 93, 71-79. Abstract

Skehel, P.A., Fabian-Fine, R., and Kandel, E.R. (2000). Mouse VAP33 is associated with the endoplasmic reticulum and microtubules. Proc. Natl. Acad. Sci. U. S. A. 97, 1101-1106. Abstract Article 
Skehel, P.A., Martin, K.C., Kandel, E.R., and Bartsch, D. (1995). A VAMP-binding protein from Aplysia required for neurotransmitter release. Science 269, 1580-1583. Abstract Article

Smith, H.E., and Ward, S. (1998). Identification of protein-protein interactions of the major sperm protein (MSP) of Caenorhabditis elegans. J. Mol. Biol. 279, 605-619. Abstract Article

Smith, J.R., and Stanfield, G.M. (2011). TRY-5 is a sperm-activating protease in Caenorhabditis elegans seminal fluid. PLoS Genet. 7, e1002375. Abstract Article

Stanfield, G.M., and Villeneuve, A.M. (2006). Regulation of sperm activation by SWM-1 is required for reproductive success of $C$. elegans males. Curr. Biol. 16, 252-263. Abstract Article

Tarr, D.E.K., and Scott, A.L. (2005a). MSP domain protein-1 from Ascaris suum and its possible role in the regulation of major sperm protein-based crawling motility. Mol. Biochem. Parasitol. 143, 165-172. Abstract Article

Tarr, D.E.K., and Scott, A.L. (2005b). MSP domain proteins. Trends Parasitol. 21, 224-231. Abstract Article

Tsuda, H., Han, S.M, Yang, Y., Tong, C., Lin, Y.Q, Mohan, K., Haueter, C., Zoghbi, A., Harati, Y., Kwan, J., et al. (2008). The amyotrophic lateral sclerosis 8 protein VAPB is cleaved, secreted, and acts as a ligand for Eph receptors. Cell 133, 963-977. Abstract Article

Varkey, J.P., Jansma, P.L., Minniti, A.N., and Ward, S. (1993). The Caenorhabditis elegans spe-6 gene is required for major sperm protein assembly and shows second site non-complementation with an unlinked deficiency. Genetics 133, 79-86. Abstract

Ward, S. (1987). Expression of sperm-specific genes during nematode spermatogenesis. Ann. N.Y. Acad. Sci. 513, 128-133. Abstract Article

Ward, S., Argon, Y., and Nelson, G.A. (1981). Sperm morphogenesis in wild-type and fertilization-defective mutants of Caenorhabditis elegans. J. Cell Biol. 91, 26-44. Abstract Article

Ward, S., Burke, D.J., Sulston, J.E., Coulson, A.R., Albertson, D.G., Ammons, D., Klass, M., and Hogan, E. (1988). Genomic organization of major sperm protein genes and pseudogenes in the nematode Caenorhabditis elegans. J. Mol. Biol. 199, 1-13. Abstract Article

Ward, S., and Carrel, J.S. (1979). Fertilization and sperm competition in the nematode Caenorhabditis elegans. Dev. Biol. 73, 304-321. Abstract Article

Ward, S., Hogan, E., and Nelson, G.A. (1983). The initiation of spermiogenesis in the nematode Caenorhabditis elegans. Dev. Biol. 98, 70-79. Abstract Article

Ward, S., and Klass, M. (1982) The location of the major sperm protein in Caenorhabditis elegans sperm and spermatocytes. Dev. Biol. 92, 203-208. Abstract Article

Washington, N.L., and Ward, S. (2006). FER-1 regulates $\mathrm{Ca}^{2+}$ mediated membrane fusion during $C$. elegans spermatogenesis. J. Cell Sci. 119, 2552-2562. Abstract Article

Whitten, S.J., and Miller, M.A. (2007). The role of gap junctions in Caenorhabditis elegans oocyte maturation and fertilization. Dev. Biol. 301, 432-446. Abstract Article

Wu, J.C., Go, A.C., Samson, M., Cintra, T., Mirsoian, S., Wu, T.F., Jow, M.M., Routman, E.J., and Chu, D.S. (2012). Sperm development and motility are regulated by PP1 phosphatases in Caenorhabditis elegans. Genetics 190, 143-157. Abstract Article

Yi, K., Buttery, S.M., Stewart, M., and Roberts, T.M. (2007). A Ser/Thr kinase required for membrane-associated assembly of the major sperm protein motility apparatus in the amoeboid sperm of Ascaris. Mol. Biol. Cell 18, 1816-1825. Abstract Article 
Yi, K., Wang, X., Emmett, M.R., Marshall, A.G., Stewart, M., and Roberts, T.M. (2009). Dephoshorylation of major sperm protein (MSP) fiber protein 3 by protein phosphatase $2 \mathrm{~A}$ during cell body retraction in the MSP-based amoeboid motility of Ascaris sperm. Mol. Biol. Cell 20,3200-3208. Abstract Article

Zajac, M., Dacanay, B., Mohler, W.A., and Wolgemuth, C.W. (2008). Depolymerization-driven flow in nematode spermatozoa relates crawling speed to size and shape. Biophys. J. 94, 3810-3823. Abstract Article

Zhao, Y., Sun, W., Zhang, P., Chi, H., Zhang, M.J., Song, C.Q., Ma, X., Shang, Y., Wang, B., Hu, Y., et al. (2012). Nematode sperm maturation triggered by protease involves sperm-secreted serine protease inhibitor (Serpin). Proc. Natl. Acad. Sci. U. S. A. 109, 1542-1547. Abstract Article

All WormBook content, except where otherwise noted, is licensed under a Creative SOMAERIGHISRESERVED Commons Attribution License. 